ORIGINAL ARTICLE

\title{
Interrelationship of Electrolyte and Vitamins Profile in Patients Experiencing Tumor Lysis Syndrome during Cancer Chemotherapy
}

\author{
KHADIJA MASTOOR ${ }^{1}$, BUSHRA SUHAIL ${ }^{1}$, ASMA INAM ${ }^{2}$, NADA AZAM ${ }^{3}$, MARIA AMJAD ${ }^{4}$, FAREEHA BASHIR ${ }^{5}$ \\ 1Department of Pharmacology, University College of Medicine \& Dentistry, University of Lahore \\ 2Department of Pharmacology, Azra Naheed Medical College, Lahore \\ 3Department of Pharmacology, Central Park Medical College, Lahore \\ 4 University Institute of Diet and Nutritional Sciences, University of Lahore \\ 5University Institute of Medical Lab Technology, University of Lahore \\ Correspondence toDr Khadija Mastoor, Email: khadija.mastoor@ucm.uol.edu.pk, Cell: 03034133109
}

\begin{abstract}
Background:Tumor lysis syndrome is a metabolic derangement which is seen in patients with malignancy and receiving drugs for cancer treatment. It can arise in children or older cancer patients and is considered life threatening. Anticancer drug therapy is most commonly used method to treat cancer.

Aim: To investigate the role of electrolytes and vitamins ( $A, C$ and $E$ ) in cancer patients suffering from tumor lysis syndrome during anticancer therapy.

Study design: Prospective clinical study

Methods: The study enrolled fifty diagnosed patients of Tumor lysis syndrome.Informed consent was taken from patients. Twenty patients, clinically healthy, age and sex-matched were selected as a control in the present study. $5 \mathrm{cc}$ blood was withdrawn from enrolled cases. The obtained samples were centrifuged at the speed of 40005000rpm for 10-15 minutes to obtain serum. The levels of Electrolytes $(\mathrm{Na}+, \mathrm{K}+)$, and Vitamins $\mathrm{A}, \mathrm{C}, \mathrm{E}$ were estimated.

Results: Study showed elevated serum levels of sodium (Na+) (28.26) in comparison withcontrol normal persons $(21.26)$ and this is significant statistically $(0.02<0.05)$. Serum Potassium levels among Tumor lysis syndrome (TLS) cases was (13.26) as observed in normal controlled persons (14.26) and results were significant statistically $(0.03<0.05)$. Vitamin A level in Tumor lysis syndrome(TLS) cases decreased outstandingly (102.20) in contrast to normal control study persons.(188.26) and this is significant statistically $(0.026<0.05)$. The values for Vitamin $E$ in Tumor lysis syndrome cases was (4.26) and in controlled normal individuals (7.26) and proved significant statistically $(0.015<0.05)$.

Conclusion: Present study showed inverse relationship between Vitamins and electrolytes in TLS. Increased level of electrolyte imbalances and decreased vitamin levels is the reason responsible for the development of tumor lysis syndrome.

Keywords: TLS, Vit A, Vit C, Vit E, Na+, $\mathrm{K}_{+}$
\end{abstract}

\section{INTRODUCTION}

Tumor lysis syndrome is basically a metabolic abnormality which manifests unexpectedly in patients with cancer receiving cancer chemotherapy ${ }^{1}$. It develops when very large number of rapidly dividing cancerous cells are disintegrated under the effect of cytotoxic therapy such as radiations or chemotherapy. This syndrome occurs mainly in patients suffering from leukemia and lymphoma. Factors which may contribute tumor lysis syndrome consists of type of cancer and cancer cell load, types of anti-tumor drugs used, and the presence of other clinical abnormalities like renal disease. Cancerous cells liberatetheir contents into the blood where they arebuild up and it becomes difficult for body to get rid of these products. Drug eliminating mechanisms cannot manage this heavy and extra burden. Cancer cells contain huge quantities of different intracellular ingredients, potassium, phosphorus and nucleic acid as compared to the normal cell. Rigorous metabolic derangement as a result of liberation of these intracellular fragments which produces deadly complications like hyperkalemia, hyperphosphatemia, hypocalcemia and

Received on 13-02-2021

Accepted on 27-06-2021 hyperuricemia. Cytokines are also liberated as a result of breakdown of malignant cell population that lead to the development of generalized inflammatory response syndrome and failure of multiple organs ${ }^{6}$.

Cancer therapy includes use of different modalities such as the use of anti-cancer agents, biological substances, corticosteroids, hormones and radiation therapy and all of these are contributing factors to the occurrence of tumor lysis syndrome. Hardly ever, TLS suddenly occurs before the institution of anticancer treatment ${ }^{2}$. Tumor lysis syndrome (TLS) is grouped intoLaboratory tumor lysis syndrome, (TLS) characterized by changes in levels of uric acid and electrolytes in blood and secondly, symptomatic Tumor lysis syndrome (TLS) constituted by the anticipatedproblems associated with these metabolic abnormalities $^{3}$. The clinical features associated with TLS usuallyappear 12-72 hours of the commencement of anticancer therapy and seizures, renal failure and abnormal heart rhythm may develop in these patients ${ }^{7}$.

The etiology of tumor lysis syndrome that produces many clinical consequences includes, elevated levels of uric acid and problems associated with it. The metabolic degradation of intracellular nucleic acid produces hyperuricemia. Purines are catabolized into uric acid through hypoxanthine and xanthine in the presence of 
xanthine oxidase ${ }^{5}$. Rapid and excessive release of the intracellular phosphate leads to hyperphosphatemia ${ }^{9}$.

Factors which contribute to the development of tumor lysis syndrome include tumor cell type and burden type of anticancer treatment used and baseline clinical conditions such as dehydration or acute renal disease ${ }^{1,2}$.Rapid proliferation of tumor cells and promptly responding tumors to the anticancer treatment, are the common sites of $\mathrm{TLS}^{8,10}$. In the development of tumor lysis syndrome, hematologic and non-hematologic carcinomas, rapidly proliferating lymphomas like Burkitt's lymphomas and acute leukemias for example acute lymphoblastic Leukemia are involved ${ }^{4}$.

Hyponatremia is described as sodium plasma levels less than $135 \mathrm{mmol} / \mathrm{L}$. It can develop promptly in about $48 \mathrm{~h}$ (acute hypernatremia). Hypernatremia iscategorized into three types: mild (130-134mEq/L), moderate (125-129 $\mathrm{mEq} / \mathrm{L})$, and severe $(<125 \mathrm{mEq} / \mathrm{L}$, depending upon its serum level ${ }^{15}$. It has been considered the largest contributing factor to tumor-related electrolyte disorder associated with TLS. Paraneoplastic syndromes for instance brain metastasis, SIAD, adrenal metastasis, and kidneymetastasis can cause decrease in blood levels of potassium ${ }^{14}$.

Hyperkalemia is characterized by elevated blood levels of potassium concentration (> $5.5 \mathrm{mEq} / \mathrm{L}$ ). Depending on potassium level, three different types are categorized: mild hyperkalemia (5.1-6.0mEq/L), moderate hyperkalemia (6.1$7.0 \mathrm{mEq} / \mathrm{L})$, and severe hyperkalemia $(>7.0 \mathrm{mEq} / \mathrm{L})$, which isalarming situation $)^{12}$. Anticancer agents, such as platinum derivatives, are highly nephrotoxic and maylead to hyperkalemia ${ }^{13}$.

Rarely, hyperkalemia may be due to only excessive intake of potassium, and it is time and again linked with furtherthreats such as diabetes mellitus, renal failure, and concurrent intake of drugs that retard potassium wasting (e.g., spironolactone, captopril, and NSAIDs) ${ }^{11}$.

Vitamins $A, C$ and $E$ are indispensable micronutrients which turns to be robust antioxidants in countless biological processes $^{18,19}$.

Chemotherapy leads to cell death indirectly by dropping levels of anti-oxidants for instance vitamins intracellularly or directly by oxidative stress ${ }^{19}$.

\section{MATERIALS AND METHODS}

Sample size: Fifty patients with Tumor Lysis syndrome (TLS) were enrolled to take part in present study from oncology unit of Jinnah Hospital Lahore.

Control group. Twenty normal persons, irrespective of age and gender, clinically healthy individuals were included as controls.

Inclusion criteria: Diagnosed cases of malignancy receivinganti-cancer treatment and without any pre-existing clinical condition before the initiation of anti-cancer treatment.

Exclusion criteria: Patients with any underlying disease before the commencement of anti-cancer treatment were not included in the study.

Sample collection: Blood from enrolled cases was taken under aseptic conditions. Permission from enrolled cases was taken from Ethical Committee before taking samples.
Sample and sampling technique: $5 \mathrm{cc}$ blood was collected with the help of needle and syringe (BD), from each enrolled patient under strict aseptic conditions, in EDTAVaccutainers and centrifuged at 4000-5000rpm for 10-15 minutes to takeserum apart.All chemicals used during research were bought from Sigma Chemical Co. (St. Louis, Mo, USA).

Parameters checked: Evaluation of electrolytes sodium $(\mathrm{Na}+)$ and potassium $(\mathrm{K}+)$ and vitamins (vit $\mathrm{A}, \mathrm{E}$ and $\mathrm{C})$ was carried out.

\section{RESULTS}

Fig-A: Serum vitamins in $\mu \mathrm{g} / \mathrm{ml}$ and electrolytes in $\mathrm{mg} / \mathrm{L}$

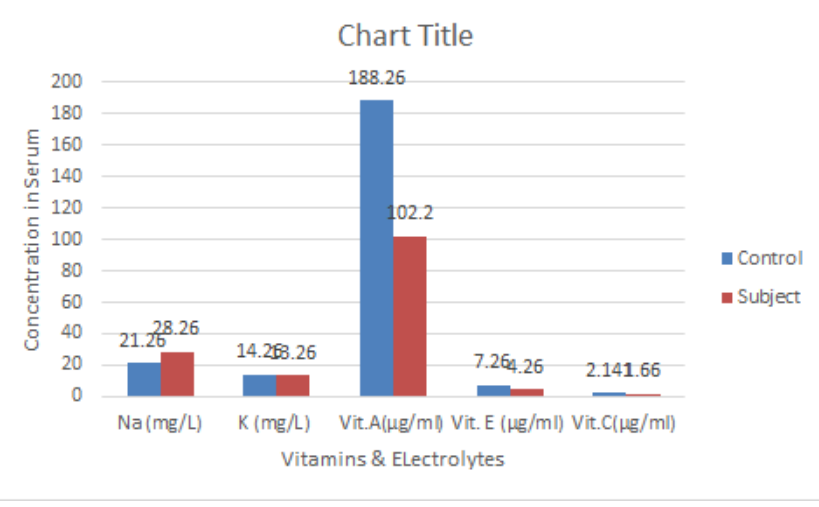

The serum electrolyte profile evaluated in cases experiencing tumor lysis showedincreased sodium $(\mathrm{Na}+)$ 28.26 in comparison with normalcontrols (21.26) andit was significant statistically $(0.02<0.05)$. The values of Potassium (K) in study enrolled cases was (13.26) in comparison with normal individuals (14.26) and proved to be significant in statistics $(0.03<0.05)$ (fig. A).During evaluation of vitamin levels, it was found that the values ofvitamin A in TLS cases decreasedamazingly (102.20) in contrast to normal controls (188.26) and was significant statistically $(0.026<0.05)$. The serum level ofVit $E$ in Tumor lysis syndrome cases was (4.26) and in normal persons (7.26) which is significant statistically $(0.015<0.05)$. Vitamin $C$ serum values declined in cases (1.66) in contrast to normal persons (2.41) which was also statistically significant $(0.016<0.05)$ (Fig. A).

\section{DISCUSSION}

Tumor lysis syndrome (TLS) is apromptly developing abnormality produced by metabolic and electrolyte imbalances that are harmful without timely identification. The formation of hyperuricemia, hyperphosphatemia, hyperkalemia and hypernatremia can cause renal and many other organ failure ${ }^{16,17}$.

Dropin vitamins level A, C and E during cancer chemotherapyindirectly spectacles boost in freeradicals within body, which specifies usefulness of treatment15 but shrunk antioxidant vitamins status may play a part in etiology of manifold diseases. Antioxidants put forth a shielding effect counter to free radicals ${ }^{18,19}$.

Large and rapidly dividing cancers of blood origin like acute leukemia, and non-Hodgkin lymphoma are the signs of high risk tumor lysis syndrome patients. 
Chemotherapy and radiation on promptly dividing cell are the major cause of tumor lysis syndrome. Those patients which required high dose chemotherapy like bone marrow transplantation are in danger to develop tumor lysis syndrome. TLS can be considered the serious complication of cancer treatment.

\section{CONCLUSION}

Present study concludes that electrolyte imbalanceand waning vitamins levels in TLS patients was due to progressive cancer chemotherapy.

Conflict of interest: Nil

\section{REFERENCES}

1. Arrambide K and RD Toto (1993). Tumor lysis syndrome. Semin Nephrol.13: 273-80.

2. Cairo MS and M Bishop (2004). Tumour lysis syndrome: new therapeutic strategies and classification. Br J Haematol. 127:311.

3. Coiffier B, A Altman, CH Pui, AYounes and MS Cairo (2008). Guidelines for the management of pediatric and adult tumor lysis syndrome: an evidence-based review. J ClinOncol. 26:2767-78.

4. Davidson MB, S Thakkar and JK Hix (2004). Pathophysiology, clinical consequences, and treatment of tumor lysis syndrome. Am J Med. 116:546-54.

5. Goldman SC, JS Holcenberg, JZ Finklestein, R Hutchinson, S Kreissman, FL Johnson, C Tou E, Harvey, E Morris, MS Cairo (2001). A randomized comparison between rasburicase and allopurinol in children with lymphoma or leukemia at high risk for tumor lysis. Blood.97(10):2998-3003.

6. Hijiya N, ML Metzger and S Pounds(2005). Severe cardiopulmonary complications consistent with systemic inflammatory response syndrome caused by leukemia cell lysis in childhood acute myelomonocytic or monocyticleukemia.Pediatr Blood Cancer.44, 63-69.
7. Hochberg J and MS Cairo (2008). Tumor lysis syndrome: Current perspective. Haematologica. 93(1):9-13.

8. Hussain K, JJ Mazza and LH Clouse (2003). Tumor lysis syndrome (TLS) following fludarabine therapy for chronic lymphocytic leukemia (CLL): Case report and review of the literature. Am J Hematol. 72:212-5.

9. Locatelli F and F Rossi (2005). Incidence and pathogenesis of tumor lysis syndrome. ContribNephrol. 147:61-8.

10. Rostom AY, El-Hussainy, A Kandil and AAllam (2000). Tumor lysis syndrome following hemi-body irradiation for metastatic breast cancer. Ann Oncol. 11..

11. Nagasaki A, Takamine W, Takasu N (2005). Severe hyperkalemia associated with " alternative" nutritional therapy. ClinNutr 24:864-5.

12. Chartyan D, Goldfarb DS (2000). Indications for hospitalization of patients with hyperkalemia. Arch intern Med. 160:1605-11.

13. Zacchia M, Stratigis $S$ (2016). Potassium: From physiology to clinical implications. Kidney Dis (basel). 2:72-9.

14. Berardi R, Santomi M, Rinaldi S, Nunzi E (2016). Risk of hyponatraemia in cancer patients treated with targeted therapies., a systematic review and meta-analysis of clinical trials. Plos One 11: e0152079.

15. Palmer BF (2003). Hyponatraemia in patients with central nervous system disease: SIADH versus CSW. Trends endocrinolMetab. 14: 182-7.

16. Verzicco I, Regolisti G,Quaini F. Electrolyte Disorders Induced by Antineoplastic Drugs. Front Oncol. 2020; 10: 779.

17. Gorkom G, Lookermans EL, Elssen $\mathrm{CH}$. The Effect of Vitamin C (Ascorbic Acid) in the Treatment of Patients with Cancer: A Systematic Review Nutrients. 2019 May; 11(5): 977

18. Shrivastava A, PradhanS. Serum vitamin A, E and C status in cervical cancer patients undergoing Concurrent ChemoRadiotherapy, an institutional study. Journal of Nutrition \& Intermediary Metabolism 18 (2019) 1001072

19. Tarlovsky VF, Rivera MA, Altamirano KA. Antioxidant supplementation has a positive effect on oxida-tive stress and hematological toxicity during oncology treatment in cervical cancer patients, Support. Care Cancer 21 (5) (2013) 13591363. 